\title{
Extended AODV For Multi-Hop WMN
}

\author{
S.Jyothsna \\ CVR College of Engineering, Department of IT, Ibrahimpatan, R.R.District, A.P., India \\ Email: jyothsna.sundaragiri@gmail.com
}

\begin{abstract}
Wireless Mesh Networks (WMN) consists of mesh routers and mesh clients, where mesh routers have minimal mobility and form the backbone of WMNs. They provide network access for both mesh and conventional clients. Existing Adhoc On Demand Distance Vector(AODV) Protocol was designed to simply yield the shortest path between a given source and its destination. This routing Protocol is not suitable for Wireless Mesh Backbone since it do not consider the impairments of wireless channels. we propose a new routing metric called Expected Transmission Count(ETX) and use this metric instead of the minimum hop count metric to find routes that result in higher throughputs. we will integrate AODV with the ETX metric. We show that ETX metric is superior to the hop count in terms of finding paths with higher throughputs.
\end{abstract}

Index Terms-WMN, AODV, Routing Protocol, Routing Metric, ETX.

\section{INTRODUCTION}

\subsection{WMN}

WMNs are comprised of two types of nodes Mesh Router and Mesh Client. Along with the routing capability for gateway/bridge functions existing in a conventional wireless router, a mesh router supports additional routing functions to provide a platform for mesh networking. Mesh routers generally have minimal mobility and their purpose is basically formation of mesh backbone for the mesh clients. The gateway/bridge functionalities in mesh routers enable the integration of WMNs with various other networks. Wireless Mesh routers enable conventional nodes equipped with wireless network interface cards (NICs) to connect directly to WMNs. Ethernet can be used to access WMNs by connecting to wireless mesh routers when wireless NICs are not available.

\subsection{Routing Metrics}

Metrics are foundations to find the high performance routing path between the source and destination. A node measures some basic elements for the link to its neighbors including packet loss, delay, hop count etc. based on these fundamental elements wireless node reassembles them into a new parameter, the radio metric, to describe the state of the link. Some popular radio metrics have been proposed recently. Per-hop RTT, Pkt Pair, ETX and ETT. ETX, proposed by De Counto, estimates the number of retransmissions needed to send unicast packets by measuring the loss rate of broadcast packets between pairs of neighboring nodes.

\section{RELATED WORK}

Protocols such as DSR and AODV were designed to simply yield the shortest path between a given source and its destination. They do not consider the effects and impairments of wireless channels.

\subsection{Our Contribution}

By considering the effect of packet losses on wireless links, we define a new metric called Expected Transmission Count (ETX) and use this metric instead of the minimum hop count metric to find routes that result in higher throughputs. Links with higher loss rates require more retransmissions to successfully transmit a packet and hence would have higher values of ETX.

In multi-hop wireless networks, communication quality depends on the route from a source to a destination. In this paper, we consider a one-dimensional multi-hop wireless network where nodes are distributed randomly and theoretically analyze the relation between communication quality and routing policy using ETX, which is the predicted number of data transmissions required to send a packet over that link, including retransmissions. First, we theoretically analyze the mean length of links, the mean number of hops, and the mean route ETX, which is the sum of the ETXs of all links in a route. We compare hop count metric and ETX in AODV, using the results of analysis and show differences between these algorithms in the route.

We provide a comparative analysis of various routing strategies that effect end to end performance in WMN. We first improve well- 
known link quality metrics and routing algorithms to better operate in wireless mesh environment.

\section{PROPOSED ALGORITHM}

The link loss rates are estimated by sending probe packets at a fixed rate of 1 Mbps. The same loss rates are assumed to hold for all data rates and packet sizes. Each node sends small broadcast probe packets every second at a fixed rate of 1 Mbps. All nodes in the vicinity that are able to receive the broadcast s keep track of the packet loss rates. For a successful transmission of a packet, both the packet and the acknowledgement in the reverse direction must be received successfully.

ETX makes the following assumptions.

1. The loss rates at $1 \mathrm{Mbps}$ are also indicative of loss rates for all data rates.

2. The loss rates for small broadcast packets are also indicative of loss rates for larger unicast packets.

To calculate ETX in real networks, each node broadcasts periodic probes to its neighbors.

The neighbors are required to echo back. ETX is estimated as the ratio between number of lost echoes and total transmitted probes.

\subsection{Route Request Initiation}

The Route Request is passing from source to destination, at every intermediate node the request is broadcasted to its neighbor nodes, until the request is reached to its destination.

When a node wants to communicate with another node it first checks its own routing table if an entry for this destination node exists. if this is not the case, the source node has to initialize a route discovery. This is done by creating a RREQ message, including the ETX to destination, the IP address of the source and the destination, the sequence numbers of both of them, as well as the broadcast ID of the RREQ. This ID and the IP address of the source node together form a unique identifier of the RREQ. When the RREQ is created the source node broadcasts it and sets a timer to wait for reply.

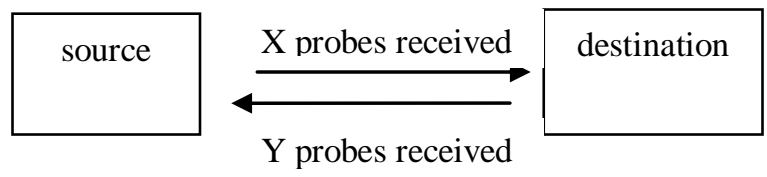

If $\mathrm{pf}$ is the probability of successful packet delivery in forward direction and $\mathrm{pr}$ is the probability in reverse direction, the probability that a packet arrives and and is acknowledged correctly is pf*pr.

The expected number of transmissions is $\mathrm{ETX}=1 / \mathrm{pf} * \mathrm{pr}$

ETX of a route is the sum of ETX for each link. The objective function for this routing metric is to pick minimum ETX path among the alternative routes.

\subsection{Route Request Completion}

The destination is sending Route Reply through reliable route, the destination choses best route by comparing ETX values from the Route Request it received from various routes.

All nodes which receive the RREQ first check by comparing the identifier of the message with identifiers of messages already received. If it is not the first time the node sees the message, it discards silently the message. If this is not the case the nodes processes the RREQ by updating its routing table with the reverse route.it creates a RREP message and unicasts it to the source node. This can be done by analyzing the reverse route for the next hop, otherwise it calculates the ETX. Updates RREQ's ETX, and Requests and then broadcasts the message to its neighbors.

\subsection{Route Maintenance}

When a route has been established, it is being maintained by the source node as long as the route is needed. Movements of nodes effect only the routes passing through this specific node and thus do not have global effects. If the source node moves while having an active session, and loses connectivity with the next hop of the route, it can rebroadcast an RREQ. Even though an intermediate station loses connectivity with its next hop it initiates an Route Error(RERR) message and broadcasts it to its precursor nodes and marks the entry of the destination in the route table as invalid, by setting the distance to infinity.

The events that must be determined are:

1. A route request: This is indicated by a locally generated packet that needs to be sent to a destination for which a valid route is not known.

2. Buffer packets during route discovery: During route discovery, packets destined for the unknown destination

should be queued. If a route is found the packets are to be sent. 
3. Update the lifetime of an active route: This is indicated by a packet being received from, sent to or forwarded to a known destination.

4. Generate a RERR if a valid route does not exist: If there is no known route to a destination, the node must send a RERR.

5. Generate a RERR during restart: After the AODV routing protocol restarts, it must send a RERR message to other nodes attempting to use it as a router.

\section{RESULtS AND ANALYSIS}

For the experiments, we choose hop-count metric to provide baseline for performance comparison along with ETX.

\subsection{Experimental setup}

Our experiments are performed using NS-2 simulations. In our experiments, we choose the 10-node scenario,20-node scenario,50-node scenario and 100-node scenario,120-node scenario,150-node scenario,180-node scenario. For simulations, we choose the distances between the nodes and their transmissions ranges in such a way that both paths are independent with the exception of sharing source and destination.

\subsection{Simulations}

NS-2 is our simulation platform and we implemented ETX with AODV routing protocol. AODV is chosen because of its suitability to the stationary scenario of wireless mesh networks. We have added probing mechanism in AODV protocols where each node broadcasts a probe packet after every $10 \mathrm{sec}$ for ETX and the receivers send back a unicast echo packet. Sending time is embedded in the probe packet. When a node receives an echo packet from a neighbor, it calculates the delay. If the delay is greater than a fix threshold i.e $10000 \mathrm{~ms}$ in our experiments, the echo packet is dropped otherwise, the node counts the dropped or lost packets to calculate ETX. ETX uses the last ten probes for computation. When a node receives routing table broadcast from a neighbor, it adds the ETX value of the link to the neighbor to the table entries and compares them with its own table.

Constant bit rate (CBR) UDP traffic is used with 1000 byte packets for various data rates form source 0 to destination 3. UDP traffic allows us to study the performance of routing metric in the simplest settings without any influence of transport protocol artifact. To implement loss in non-preferred path, a node on the path is made to drop random packets among all it receives according to specific probability of loss. Each result is averaged over 10 simulation runs and error bars in figures show $95 \%$ confidence interval.

We got expected results for ETX performance under lower data rate, but with higher data rates ETX performance was comparatively low. The results are shown interestingly, when one path become much worse than the other, ETX has better throughput, i.e., lower loss rate than hop-count. Hop-count always has a lower buffer overflow loss than ETX, because it does not use probes. However, hop-count does not distinguish between higher or lower loss paths. As a consequence, when losses in one path becomes higher, packets dropped due to bad channels become more significant than packets dropped due to buffer overflow and overall performance of hop-count metric is worse than ETX.

$$
\text { No.of Nodes vs throughput }
$$

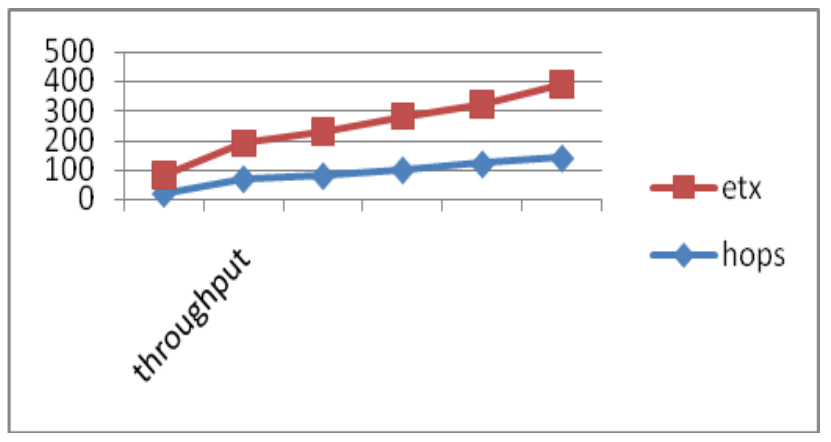

By analyzing the results, we can observe the throughput is more where ETX as the metric. Because, by calculating the ETX values on the links, nodes can chose the lower ETX paths i.e reliable links.

\section{CONCLUSION}

\section{Summary}

In this paper, a survey of routing metric carried out, we proposed and implemented ETX enhancement over well known AODV routing protocol. The prerequisites that a multi-radio multi-channel mechanism must have are also studied. Steps and method to be used for new metrics are discussed.

Based on our experiments, we observed our proposed Routing Protocol is performing better 
than existing AODV, since ETX chooses paths based link quality rather than number of hops. ETX finds routes that result in higher throughputs. We show that ETX metric is superior to the hop count metric in terms of finding paths with higher throughputs.

ETX chooses links with higher delivery ratio. Buffer overflow loss in ETX is in general more than that of hop count, which has no probing mechanism.

\section{REFERENCES}

[1] R.Draves, J.Padhye, and B.Zill, "Comparison of routing metrics for static multi-hop wireless networks," in SIGCOMM '04: Proceedings of the 2004 conference on Applications, technologies, architectures, and protocols for computer communications, 2004, pp.133-144.

[2] D.S.J.D. Couto, D. Aguayo, J.Bicket, and R. Morris, "A high throughput path metric for multi-hop wireless routing," Wirel. Netw., vol. 11, no. 4, pp. 419-434, 2005.

[3] I.F. Akyildiz and W. Xudong, "A survey on wireless mesh networks," IEEE communications Magazine, vol. 43, pp. 23-30, 2005.

[4] W.S.,B. Ishibashi, R. Boulaba, and D. Meddour, "Performance study of wireless mesh networks routing metrics ," in Proc. IEEE/ACS AICCSA 2008, March-April 2008,pp. 1100-1106.

[5] S.Misra et al., "Guide to Wireless Mesh Networks" Springler-Verlag London Limited 2009

[6] C. Perkins, E.Belding-Royer, S.Das. Ad hoc On-Demand Distance Vector (AODV) Routing, RFC 3561.

[7] "The Network Simulator-ns-2." from http://www.isi.edu/nsnam/ns. 